\title{
El feminismo, el género y la profesionalización del trabajo social en Colombia (1936-2004)
}

\author{
Ambar Oriana Serna-Lombo \\ Magister en Trabajo Social. Trabajadora Social \\ Universidad Nacional de Colombia. Bogotá, Colombia \\ https://orcid.org/0000-0003-2180-1184 • aosernal@unal.edu.co
}

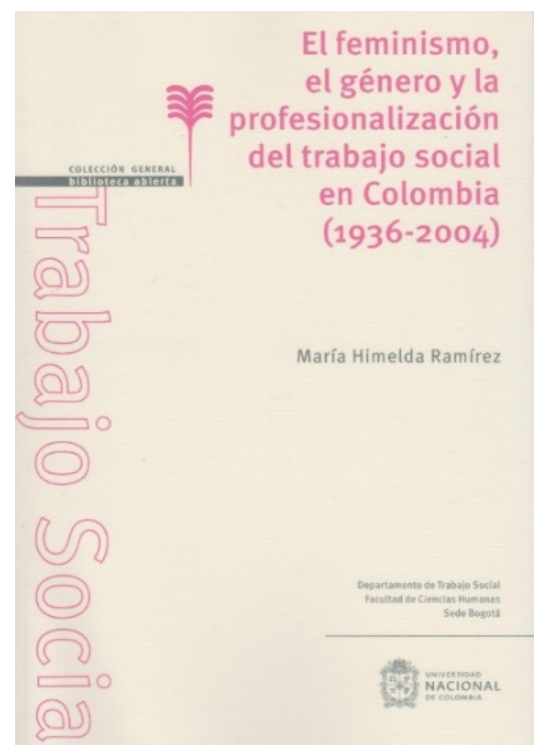

\author{
Ramírez, María Himelda. 2020. \\ El feminismo, el género y la \\ profesionalización del trabajo social en \\ Colombia (1936-2004). \\ Bogotá. Universidad Nacional de \\ Colombia. Facultad de Ciencias \\ Humanas. Departamento de Trabajo \\ Social. \\ 212 p. \\ ISBN 978-958-794-145-6.
}

El feminismo, el género y la profesionalización del trabajo social en Colombia, propone un recorrido por alrededor de ocho décadas del proceso de profesionalización del trabajo social en nuestro país, con especial atención en el proceso de inserción de los programas de formación en asistencia social, servicio social y posteriormente, Trabajo Social en la educación superior. El amplio periodo en el que discurre la investigación y la variedad de fuentes históricas que la constituyen permiten, en primera instancia, avizorar la relevancia y novedad de este libro que supone un esfuerzo importante por recuperar -desde sus

Recibido: 30/08/2020 | Aprobado: 06/11/2020 | Publicado: 01/01/2021

\section{¿Cómo citar este artículo? / How to quote this article?}

Serna-Lombo, A. O (2021). El feminismo, el género y la profesionalización del trabajo social en Colombia (1936-2004). Prospectiva. Revista de Trabajo Social e intervención social, (31), 469-474. doi: 10.25100/prts.v0i31.10573. 
protagonistas- documentar y sistematizar la historia de esta profesión y a la vez, parte de las tensiones y posibilidades del acceso de las mujeres a la educación superior en Colombia. Estos decenios son transitados desde la perspectiva de la historia de las mujeres, develando desde una perspectiva crítica, entre otros asuntos, las posibilidades de acceso a lo público que supuso para las mujeres en la primera mitad del siglo $\mathrm{XX}$, formarse y ejercer una profesión que incluso fue respaldada desde posturas conservadoras, al considerarse compatible con las labores del hogar; y de otra parte, revela también los obstáculos y la subordinación entre las ciencias sociales que supuso el proceso de profesionalización de una profesión calificada "femenina" como el Trabajo Social. En esta línea Ramírez (2020) afirma:

El trabajo permite vislumbrar las formas como operó la diferencia de género en el acceso a la educación superior en Colombia, así como también, en las estrategias de quienes optaron por la formación en asistencia social, servicio social y trabajo social para incidir en el cambio en sus propias vidas, así como en las condiciones de vida de los sectores populares de la población urbana en expansión, afectados por la pobreza y la exclusión. (p. 21)

Las obras, sin duda adquieren la impronta de quienes la forjan, y en este caso, la relación entre este libro y su autora, María Himelda Ramírez, es manifiesta. Doctora en Historia de América de la Universidad de Barcelona, Magíster en Historia y Licenciada en Trabajo Social de la Universidad Nacional de Colombia. Este libro conjuga su formación y experiencia, en el análisis de este proceso histórico del trabajo social, del que ella misma fue participe en parte del periodo estudiado, pues cursó trabajo social en la Universidad Nacional de Colombia entre 1969 y 1974; desde 1976 hasta 2012 estuvo vinculada como docente de pregrado de Trabajo Social, docente de los posgrados de la escuela de Estudios de Género y la Maestría el Trabajo Social con énfasis en familia y redes sociales, de la que también fue coordinadora. Así, su autora, reconstruye una historia de la que ha sido testigo y protagonista privilegiada, como mujer colombiana, estudiante, egresada y docente de trabajo social, feminista y cofundadora del Grupo Mujer y Sociedad de la Universidad Nacional de Colombia.

Este libro es resultado de un proceso de investigación, que se ubica dentro de los intereses investigativos de larga data de la autora, quien pertenece al Grupo de Investigación de la Asistencia Social, la Beneficencia y el Trabajo Social de la Universidad Nacional de Colombia. Para su construcción retoma una amplia variedad de fuentes históricas, entre las que se destacan las entrevistas -algunas publicadas y otras inéditas- a egresadas de los programas de Trabajo Social en sus diversos momentos de profesionalización, es decir, desde las Escuelas de Servicio Social adscritas al Colegio Mayor de Nuestra Señora del Rosario en Bogotá o la Universidad Pontifica Bolivariana en Medellín, hasta egresadas de Trabajo Social de la Universidad Industrial de Santander y la Universidad Nacional. De otra parte, es de destacar el análisis de tesis de grado, una revisión de algunas de las publicaciones periódicas publicadas por las egresadas, que señalan sus 
intereses, desarrollos profesionales e incluso posturas políticas a favor de sus congéneres. A estas fuentes se suma el análisis de documentos de archivo y de naturaleza jurídica, que señalan los procesos de transición y transformación, tanto del proceso de profesionalización del trabajo social en diversas unidades académicas pasando por sus currículos y las intencionalidades en la formación, como los avances en la construcción de la Escuela de estudios de género y sus estudios pos graduales que se adelantaron en la Universidad Nacional. De otra parte, se recorre la amplia producción académica de varios profesoras y profesores pioneros de trabajo social y la Escuela de estudios de género en la Universidad Nacional.

La obra se estructura en cuatro capítulos que parten de una importante revisión sobre el proceso de institucionalización del servicio social en la década de los años treinta en varias ciudades del país, para progresivamente centrar sus análisis en varios de los desarrollos específicos del Trabajo Social en la Universidad Nacional de Colombia y algunas de sus egresadas enmarcadas en el feminismo académico de finales del siglo XX e inicios del siglo XXI, en conexión con el discurrir del feminismo y los estudios de género latinoamericanos.

El primer capítulo denominado "La institucionalización del servicio social católico" se refiere al periodo comprendido entre 1936 y 1958, presenta los desarrollos dispares, así como las convergencias de los tres procesos educativos que podrían considerarse pioneros del servicio social en Colombia: la Escuela de Servicio Social anexa al Colegio Mayor de Nuestra Señora del Rosario, la Escuela de Servicio Social adscrita a la Normal de Señoritas de Antioquia y los Colegios Mayores de Cultura Femenina desplegados en varias ciudades del país, con un especial énfasis en las dos primeras. Este capítulo pone de presente las intersecciones entre el momento histórico que vivieron las pioneras, quienes experimentaron el tránsito entre la república liberal y la restauración conservadora, en pleno proceso de urbanización e industrialización en Bogotá y Medellín en el que se evidenciaba una amplia complejidad de problemas sociales propios de estos procesos, que inspiraron a mujeres jóvenes de élites urbanas procedentes de hogares católicos a organizarse y formarse para la atención de los mismos.

Inicialmente movidas por ideas de la caridad católica, y pese a que sus planes de estudios estaban fuertemente orientados por la religión, se encontraron progresivamente en sus desarrollos profesionales y publicaciones, movilizadas por ideas de la modernidad liberal, asociadas a la justicia social y una militancia en el movimiento sufragista, que reclamaba el pleno ejercicio de la ciudadanía de las mujeres. Este capítulo pese a su amplio recorrido, deja en punta el análisis respecto a los Colegios Mayores de Cultura Femenina, y señala las posibilidades que supone el desarrollo de futuras investigaciones alrededor de estas instituciones de carácter público y su relevancia para el posterior avance del trabajo social en ciudades como Cartagena y Popayán. 
Serna-Lombo

Por su parte, el segundo capítulo titulado "Hacia la laicidad en la formación en trabajo social en las universidades públicas. El caso de la Universidad Nacional de Colombia", presenta el proceso de transición del programa de Trabajo Social del Colegio Mayor de Cultura Femenina de Cundinamarca a la Facultad de Sociología de la Universidad Nacional. Se observan algunas de las tensiones que implicó este tránsito, enmarcado en la subordinación de campos de conocimiento entre la sociología y el trabajo social, y la ausencia de autonomía de este último, que se materializó por ejemplo, en la imposibilidad de desarrollar investigación independiente hasta 1985 cuando pudo constituirse el Departamento de Trabajo Social.

Este capítulo propone además, un interesante recorrido por algunas de las obras destacadas e intereses investigativos de algunos de los primeros docentes del programa como: María Cristina Salazar, Virginia Gutiérrez de Pineda, Álvaro Villar y Florence Thomas, quienes empiezan a trazar derroteros sobre lo que serían los posteriores desarrollos del Departamento y sus egresadas, comprometidas en escenarios de práctica en sectores populares y análisis críticos sobre las relaciones familiares y de pareja, señalando la organización patriarcal de algunas familias y las diferencias en la construcción de subjetividades femeninas y masculinas. El capítulo, a continuación, se aproxima a los efectos que se evidenciaron en el proceso de formación del Departamento, derivados del proceso de reconceptualización que en línea con los desarrollos del pensamiento crítico latinoamericano de mediados de los años sesenta, cuestionaron los métodos y procesos de intervención clásicos del trabajo social. Se concluye este capítulo señalando avances del Departamento en el desarrollo de investigaciones desde el feminismo o los estudios de género, que logran posicionar esta categoría, para analizar por ejemplo las maternidades, paternidades, la violencia en las relaciones familiares, estudios liderados, entre otras, por María Eugenia Martínez, María Himelda Ramírez, Yolanda Puyana, docentes de Trabajo Social y fundadoras del grupo Mujer y Sociedad, explicitando con mayor contundencia la relación entre el devenir del trabajo social y el feminismo en Colombia.

“Anotaciones sobre los estudios de mujer y género en la segunda mitad del siglo XX: conexiones latinoamericanas: años 70 y $80^{\prime \prime}$, así se titula el tercer capítulo, que introduce el contexto político social, académico y organizativo que posibilita la emergencia de estudios sobre mujer y género en diversos escenarios académicos de Latinoamérica y, concretamente, en la Universidad Nacional el programa de estudios de Género, Mujer y Desarrollo. Entre varios de estos elementos de contexto, se señala la relevancia de la Comisión Económica para América Latina y el Caribe-CEPAL-, que desde su División de Asuntos de Género evidencia en sus informes e indicadores las condiciones diferenciales y de discriminación de las mujeres en la región, posicionando categorías analíticas como la doble jornada de trabajo. Estas décadas marcadas por las dictaduras en la región, también supusieron escenarios de movilización y organización social, en los que fueron claves los Encuentros Feministas Latinoamericanos y del Caribe, que posibilitaron la interlocución de diversos 
feminismos que posicionaron nuevas formas de interpretar la vida de las mujeres. Este recorrido evidencia el rol de varias docentes y egresadas de trabajo social, como mujeres que posicionadas desde el feminismo académico, promovieron estos estudios desde organizaciones sociales como ONG'S y desde la academia.

Por último, "La institucionalización de los Estudios de Género de la Universidad Nacional y la consolidación de la Escuela" concluye este recorrido en el periodo histórico de 1986 a 2004 en el que se consolida el Grupo Mujer y Sociedad, como se señaló previamente, fundado entre otras por trabajadoras sociales, que fueron fortaleciendo reflexiones sobre el feminismo y la diferencia, ubicadas en las singularidades del contexto colombiano, signado de forma contundente por la violencia. Este apartado del capítulo presenta, además, un interesante recorrido por algunas publicaciones periódicas promovidas por colectivos feministas en diversas regiones del país, denotando el ambiente favorable al pensamiento crítico feminista que han supuesto las últimas décadas. Este capítulo expone los debates y desarrollos que permitieron transitar al grupo Mujer y Sociedad, de la organización del Fondo de Documentación Mujer y Género, al desarrollo de los posgrados -especialización y maestría- así como la configuración de una publicación de larga trayectoria del grupo "En Otras palabras...", que hasta hoy permanecen como legado vigente en la Universidad Nacional. Se concluye este acápite con anotaciones sobre el pensamiento y la perspectiva de género en los estudios de familia, de las que podrían derivarse posteriores investigaciones.

En resumen, este libro además de muy recomendado por el amplio recorrido que propone, la riqueza de fuentes a las que recurre, es de suma vigencia al enmarcarse dentro del movimiento que se viene gestando en algunas unidades académicas latinoamericanas y europeas -principalmente españolas y francesas- de revalorización de la historia del trabajo social y de las pioneras de Trabajo Social, y que ha llevado entre otros a la conformación de un Grupo Interuniversitario de Investigadores en Trabajo social, grupo con el que la autora ha establecido intercambios.

El título del libro presenta tres palabras clave: profesionalización, feminismo y género, que anticipan ejes analíticos que se entrelazan en el proceso de constitución de esta obra, pues se constata en su recorrido histórico, que referirse al trabajo social en Colombia, implica hacer un recorrido sobre una "profesión femenina" y de allí, la pertinencia de la categoría género para recorrer esta historia desde las pioneras asistentes sociales, hasta las egresadas y docentes de trabajo social, que se han ubicado desde el feminismo promoviendo intensas reflexiones y desarrollos académicos y en política pública a favor de las mujeres, en diversas condiciones. Así, el pensamiento feminista permite interpelar e interpretar la historia de las mujeres colombianas en diversas épocas y en particular las de aquellas que han accedido a la formación en asistencia social, servicio social o, finalmente, Trabajo Social. Estos ejes analíticos abren múltiples posibilidades de aproximación al libro, que puede ser 
Serna-Lombo

leído desde la historia del trabajo social, desde la historia del feminismo colombiano o desde la historia de las mujeres en Colombia y su acceso a la educación superior, denotando la rigurosidad que lo constituye.

\section{Referencias bibliográficas}

Ramírez, M. H. (2020). El feminismo, el género y la profesionalización del trabajo social en Colombia (1936-2004). Bogotá. Universidad Nacional de Colombia. Facultad de Ciencias Humanas. Departamento de Trabajo Social. 


\section{OTROS ARTÍCULOS DE PROSPECTIVA No. 31 DE 2021}

\section{PRESENTACIÓN}

Presentación. Reflexiones sobre desafios al publicar sistematizaciones

Rosa María Cifuentes-Gil

\section{EDITORIAL}

Reflexiones sobre Trabajo Social: aportes de la Sistematización

María Rocío Cifuentes-Patiño

\section{ARTÍCULOS}

Hacer lo que se sabe, pensar lo que se hace. La sistematización como modalidad investigativa Alfonso Torres-Carrillo

Aportes y desafios de la Sistematización de experiencias en el Trabajo Social y la extensión crítica. Apuntes y reflexiones desde la perspectiva de la Educación Popular

María Rosa Goldar

Valeria Chiavetta

La sistematización en Trabajo Social y la epistemología feminista del punto de vista. Diálogos sobre la producción de conocimiento sustentada en experiencias

Ruth Noemí Parola

María Florencia Linardelli

La Sistematización investigativa de las experiencias: del baile de los que sobran a la fiesta de los que faltan

María Belén Ortega-Senet

Sistematización y Trabajo Social en Chile. El largo y sinuoso camino

Patricia Lorena Castañeda-Meneses

Ana María Salamé-Coulon
Sentipensar la pandemia COVID-19 desde la sistematización de la experiencia en Trabajo Social: reflexiones del profesor Oscar Jara Holliday

Elia Sepúlveda-Hernández

La sistematización de experiencias, una investigación social cualitativa que potencia buenas prácticas de convivencia y gobierno. La experiencia de un conjunto residencial multifamiliar en Cali, Colombia Martha Lucia Echeverry-Velásquez Manuela Prada-Dávila

Construcción de subjetividades epistemológicaspolíticas de profesoras y profesores de Investigación social en una universidad privada y confesional en Bogotá

Giovanni Mora-Lemus

Sistematización de la experiencia Reconocimiento de los derechos humanos del adulto mayor en dos familias residentes en Cali y Valledupar (Colombia)

Lina María Cuello-Lacouture

Jimena del Pilar Jaramillo-Jaramillo

La memoria transformadora como estrategia de intervención profesional en los procesos de reconciliación social: comprensión a partir de mujeres campesinas, excombatientes y jóvenes en Manizales, Colombia

Yeimmy Stephania Corredor-Sotelo

Juliana Fuertes-Fuertes

Sistematización de una estrategia de educación informal implementada en personas privadas de la libertad en el establecimiento penitenciario de mediana seguridad y carcelario de Barranquilla, Colombia

Rafael Humberto Herrera-Mercado Rafael Alberto Zambrano-Vanegas 
Aportes significativos del proceso de intervención comunitaria con la Escuela Popular de Comunicación Alternativa Jaime Garzón de la ciudad de Cúcuta, Colombia

Carlos Lasso-Urbano

La sistematización de la intervención como metodología de investigación en Trabajo Social. Importancia práctica y teórica de la fase de recogida de datos en la intervención social según experiencia del Programa de Apoyo a las Familias en Zaragoza, España

Elisa Esteban-Carbonell

Nuria Del Olmo-Vicén

Papel de la sistematización de experiencias en los procesos de evaluación de intervenciones de salud pública en la Comuna Saludable por la Paz, Cali - Colombia

Jenny Faisury Peña-Varón

Paola Andrea Marín-Velásquez

Janeth Mosquera-Becerra

Experiencia de intervención social en hogares comunitarios integrales del barrio Alfonso Bonilla Aragón, Cali - Colombia

Julián Alexander Montaño-Cárdenas

Las políticas sociales y el gobierno de la "población indígena". Estrategias y regulaciones en el multiculturalismo chileno

Rodrigo Agustín Navarrete-Saavedra

Representaciones sociales sobre estilo de autoridad y tipos de interacción en cuidadores de residencias de protección infantil en Chile

Marcelo Gallegos-Fuentes
Carmen Gloria Jarpa-Arriagada

Reflexiones sobre inseguridad social y cuestiones penales. Una respuesta estratégica a partir de experiencias de cooperativismo con ex detenidos en Argentina

Analia Elizabeth Otero

Yael Yanina Barrera

Desarrollo y salud: la emergencia de un nuevo paradigma

Jesús María Sánchez-Ordóñez

Trabajo Social en ejercicio libre: la perspectiva profesional en España

Paula Frieiro-Padín

Tamara Fernández-Arias

Rubén González-Rodríguez

\section{RESEÑAS}

Social Work and the City: Urban Themes in 21stCentury Social Work

Felipe Saravia-Cortés

Respuestas del Trabajo Social ante emergencias sociales y problemáticas sociales complejas de México y España

Felipe Saravia-Cortés

El feminismo, el género y la profesionalización del trabajo social en Colombia (1936-2004)

Ambar Oriana Serna-Lombo

El puño invisible. Arte, revolución y un siglo de cambios culturales

Carlos Arturo Robledo-Marín

\section{PROSPECTIVA}

No. $31 \bullet$ ene.-jun. 2021

e-ISSN: 2389-993X • Universidad del Valle 